\title{
DESIGN OF TRANSPORTATION SYSTEMS WITH A DECISION AID TOOL
}

\author{
Roland Hellberg, Linjegods AS, Norway \\ Heinz Michael Winkels, IBIES FH Dormund
}

This paper describes a case study of the design and evaluation of a terminal based transportation system for groupage with a high customer service level, performed by Linjegods A.S. in Norway. The project has been supported by a tailor-made decision aid tool (TERMLOC) that provides the best location of terminals under time-restrictions with respect to lowest total cost for collection, traffic-lines, distribution and handling.

In a terminal based transportation system the costs are mainly constituted by distribution/collection activities and the terminals. The quality of a groupage system mainly depends on collection and delivery time. In this sense the quality will increase with a great number of terminals and short distances to the various market areas since this implies short collecting and distribution time. On the other hand, a great number of terminals implies high cost for running these and high transportation costs for small volumes transported to each destination. That is where the multiple criteria conflict between cost minimisation and service level maximisation begins.

The problem is to find the localisation of terminals rendering the best and cheapest geographical covering. The solution includes an evaluation of the geographical covering and a comparison with the total cost for collection, terminal-handling, traffic-lines and distribution. It also contains an cvaluation of alternative network structures (traffic-lines). TERMLOC is a tool based on a PC or UNIX database with additional simulation and optimisation modules. The data base is feed with all consignment flows during one year from zip code area to zip code area and the basic data of the transportation company (Linjegods) network structure.

The generation of free locations is based on an heuristic cluster analysis, where the locations are chosen in the order of the highest potential amount of collected and distributed goods. The reachable areas assigned to a specific terminal depend on the time restriction for collection and distribution. In TERMLOC the trafficlines schedule can be manipulated interactively with respect to the use of transfer terminals and with respect to the mode of transport. After each manipulation the algorithm recalculates the container densities, the number of empty containers, and the cost of the traffic-line, etc.

Based on the existing terminal structure and with completely free locations, 21 different alternatives with connected traffic-line network and cost structure were derived. With respect to provided service level and the cost level the preferred new structure turned out. The solution will provide nearly the same service as present situation but with $12-15$ MNOK lower cost. (The cost minimum is 20-25 MNOK, but then the service level in some areas will be lower.) 